Article

\title{
Optimization of Plastic Scintillator for Detection of Gamma-Rays: Simulation and Experimental Study
}

\author{
Sujung Min ${ }^{1,2}$, Youngsu Kim ${ }^{2}$, Kwang-Hoon Ko ${ }^{3}$, Bumkyung Seo ${ }^{2}$, JaeHak Cheong ${ }^{1, * \mathbb{C}}$, Changhyun Roh ${ }^{2,4, *(\mathbb{D})}$ \\ and Sangbum Hong ${ }^{2, *}$ \\ 1 Department of Nuclear Engineering, Kyung-Hee University, Yongin-si 17104, Korea; sjmin@kaeri.re.kr \\ 2 Decommissioning Technology Research Division, Korea Atomic Energy Research Institute, \\ Daejeon 34057, Korea; yk@kaeri.re.kr (Y.K.); bumja@kaeri.re.kr (B.S.) \\ 3 Laser Application Research Team, Decommissioning Technology Research Division, Korea Atomic Energy \\ Research Institute, Daejeon 34057, Korea; khko@kaeri.re.kr \\ 4 Quantum Energy Chemical Engineering, University of Science and Technology (UST), 217 Gajeong-ro, \\ Daejeon 34113, Korea \\ * Correspondence: jhcheong@khu.ac.kr (J.C.); chroh@kaeri.re.kr (C.R.); sbhong@kaeri.re.kr (S.H.)
}

check for updates

Citation: Min, S.; Kim, Y.; Ko, K.-H.; Seo, B.; Cheong, J.; Roh, C.; Hong, S. Optimization of Plastic Scintillator for Detection of Gamma-Rays: Simulation and Experimental Study. Chemosensors 2021, 9, 239. https://doi.org/ $10.3390 /$ chemosensors 9090239

Academic Editor: Steven W. Buckner

Received: 14 July 2021

Accepted: 24 August 2021

Published: 25 August 2021

Publisher's Note: MDPI stays neutral with regard to jurisdictional claims in published maps and institutional affiliations.

\begin{abstract}
Plastic scintillators are widely used in various radiation measurement applications, and the use of plastic scintillators for nuclear applications including decommissioning, such as gamma-ray detection and measurement, is an important concern. With regard to efficient and effective gammaray detection, the optimization for thickness of plastic scintillator is strongly needed. Here, we elucidate optimization of the thickness of high-performance plastic scintillator using high atomic number material. Moreover, the EJ-200 of commercial plastic scintillators with the same thickness was compared. Two computational simulation codes (MCNP, GEANT4) were used for thickness optimization and were compared with experimental results to verify data obtained by computational simulation. From the obtained results, it was confirmed that the difference in total counts was less than $10 \%$ in the thickness of the scintillator of $50 \mathrm{~mm}$ or more, which means optimized thickness for high efficiency gamma-ray detection such as radioactive ${ }^{137} \mathrm{Cs}$ and ${ }^{60} \mathrm{CO}$. Finally, simulated results, along with experimental data, were discussed in this study. The results of this study can be used as basic data for optimizing the thickness of plastic scintillators using high atomic number elements for radiation detection and monitoring.
\end{abstract}

Keywords: plastic scintillator; thickness; simulation; MCNP; GEANT4; total counts

\section{Introduction}

Since the plastic scintillator was first developed by Schorr and Torne in 1950 [1], it has been widely used in nuclear physics, high energy physics fields, and industrial fields such as thickness and density measurement. In addition, Buck and Swank conducted a study [2] on the performance of organic compounds on polystyrene and polyvinyl toluene, and Hayes conducted a study on a new scintillation material. "Aromatic substituted oxazole" is a long-chain structure composed of simple aromatic rings, and these materials are dissolved in an organic solvent, and various types of compounds have been successfully demonstrated in liquid and plastic scintillators [3,4]. Recently, research on the development of high-efficiency plastic detectors sensitive to radiation by adding a high atomic number material to a plastic matrix has been conducted [5].

A unique characteristic of zero-dimensional semiconductor crystals called quantum dots is that it is possible to control the carrier confinement effect and the band gap (eV). For example, CdS is one of the II-VI semiconductors and has a bandgap of about $2.42 \mathrm{eV}$. Recently, core/shell type composite quantum dots have been studied in optoelectronic applications [6-8]. In particular, it has been reported that quantum dots covered with inorganic materials such as $\mathrm{CdSe} / \mathrm{ZnS}, \mathrm{CdS} / \mathrm{ZnS}$, and CdSe/CdS have improved emission 
efficiency. Quantum dots with a core/shell structure can change their physical/optical properties through particle size or bandgap control. Therefore, quantum dots have the advantage of forming stable scintillation [6-13].

Scintillators can be broadly classified into organic and inorganic materials. Inorganic scintillators based on high atomic number materials are suitable for gamma-ray detection and spectroscopy. However, their use is limited due to inefficiency, non-uniform optical properties, long decay time, etc. Research on economical and reliable radiation detectors is being actively conducted not only in the medical field and environmental radiation monitoring, but also in nuclear material detection and decommissioning of nuclear facilities. Although plastic scintillators are used in large-volume detection systems, their stopping power is low due to their low atomic number material, so the performance for high-energy gamma-ray detection is low [5,14-17]. In nuclear-related facilities, radioactivity must be monitored regularly. Additionally, radiological characteristics evaluation is essential when operating and dismantling nuclear facilities. In terms of radiation management, radioactive waste generated from nuclear facilities requires thorough radiological measurement and evaluation because there is a possibility of radiation exposure of workers and the release of radioactive materials into the environment during decommissioning. The scintillation detector widely used for in situ measurements is one of the most representative methods of indirect ionization and consists of a scintillator and an optical sensor [18-22]. The scintillator interacts with radiation such as gamma-rays and then emits light in the UV or visible region, and the photosensor converts the light into an electrical signal. The fluorescence generation of organic scintillators is caused by energy transfer of molecules. After the molecules in the stable state are excited by radiation, the molecules return to the stable state by consuming energy in the form of lattice vibrations or heat. Fluorescence is generated in the process of returning to the stable state from the excited state [23]. The three basic characteristics of a scintillation detector can be summarized as interaction efficiency with radiation, photon generation (scintillation efficiency), and decay time.

High-resolution detection systems often use plastic-based detectors as secondary detectors for secondary signal detection, but these systems are uneconomical. Therefore, there is a need to develop a new plastic scintillator material that can provide gamma spectroscopy by reinforcing the existing plastic radiation measurement system. A plastic detector for gamma spectroscopy should have a high effective atomic number and a high light yield. For this purpose, when doping a new material, a plastic size optimization step is required to efficiently deploy the plastic scintillator [24,25]. Interaction efficiency is determined by a high effective atomic number and density and the thickness of a single crystal to increase gamma-ray absorption. Light yield is a unique property of each scintillation material. Light yield is expressed as the number of photons generated per $\mathrm{MeV}$. The light yield and the quantum efficiency of the photosensor determine the energy resolution of the detector. The measured quantity collected at the anode by gamma-rays, that is, the total number of photoelectrons, is proportional to the absolute number of photons as shown in Equation (1).

$$
N_{p h e}(E) \propto N_{p h}(E) \times \eta_{L} \times Q . E(\lambda) \times \varepsilon
$$

Here, $N_{\text {phe }}(E)$ is the number of photoelectrons collected in the anode, $N_{p h}(E)$ is the number of photons per energy unit generated by the scintillator, $\eta_{L}$ is the light collection efficiency, Q.E $\lambda(\lambda)$ is the quantum efficiency, and $\varepsilon$ is the efficiency of photoelectrons or electron-hole pair collections.

The number of photons per energy $(\mathrm{MeV})$ generated by gamma-rays depends on the gamma-ray energy, typically lower in the low-energy region below $100 \mathrm{keV}$ and peaking at $100-200 \mathrm{keV}$. Commonly, the photo-electron yield and electron-hole pair yield are corrected for the quantum efficiency of the detector and to obtain the absolute photon yield [26,27].

In this study, MCNP and GEANT4 codes were used to optimize the thickness of a plastic scintillator doped with CdS/ZnS, a quantum dot with characteristics such as a wide absorption wavelength band, a narrow emission wavelength band, and high lumi- 
nescence efficiency. MCNP and GEANT4 codes can simulate particle interactions related to neutrons, photons, and electrons, so they are used in the fields of radiation dosimetry, radiation shielding, radiography and medical physics, and radiation detector design and analysis [28-33]. In addition to optimizing the thickness using codes, a plastic scintillator doped with $\mathrm{CdS} / \mathrm{ZnS}$ was fabricated to evaluate basic optical properties. Additionally, measurement tests were performed on ${ }^{137} \mathrm{Cs}$ and ${ }^{60} \mathrm{Co}$ sources with plastic scintillators of 30 and $50 \mathrm{~mm}$ thickness. Measurement test results were compared and analyzed with simulation results.

\section{Materials and Methods}

\subsection{Fabrication of Plastic Scintillator and Spectroscopic Analysis}

$\mathrm{CdS} / \mathrm{ZnS}$ quantum dots of high atomic number were added to a styrene-based plastic scintillator, and a scintillator with a diameter of $50 \mathrm{~mm}$, thickness of $30 \mathrm{~mm}$, and $50 \mathrm{~mm}$ was fabricated through a thermal polymerization process. The materials and amounts used to manufacture the plastic are shown in Table 1 . The primary solvent converts the particle kinetic energy into excitation energy, and the primary fluorescent material, 2,5diphenyloxazole (PPO), converts the excitation energy into light. The secondary fluorescent material, POPOP (1,4 di[2-(5 phenyloxazolyl)]benzene), acts as a wavelength shifter, and the added $\mathrm{CdS} / \mathrm{ZnS}$ quantum dots not only act as a wavelength shifter but also interact with incident photons. Quantum dots increase the interaction rate with photons and affect measurement efficiency. The amounts of primary and secondary solvents and quantum dots added to the host material styrene were determined based on the results of previous studies [34-36], and PPO (0.4 wt\%), POPOP (0.01 wt\%), and CdS/ZnS (0.2 wt \%) were added. The polymerization process was performed, cutting and polishing the surface to increase the transparency as per our previous protocol $[35,36]$. For spectroscopic analysis, a handmade apparatus was set up for measurements of absorption and transmission from a fabricated plastic scintillator. Furthermore, a specific sample compartment module of different plastic scintillator-based thicknesses was designed for photoluminescence analysis.

Table 1. Content and properties of materials added in this study.

\begin{tabular}{ccccc}
\hline & Material & Quantity & Bandgap (eV) & Emission Wavelength (nm) \\
\hline 1 & Styrene & $>99 w \mathrm{wt} \%$ & - & $300-350[37]$ \\
2 & PPO & $0.4 \mathrm{wt} \%$ & - & $340-380[38]$ \\
3 & POPOP & $0.01 \mathrm{wt} \%$ & - & $400-450[39]$ \\
4 & CdS $/ Z n S$ & $0.2 \mathrm{wt} \%$ & $3.3[39]$ & $424-470[40]$ \\
\hline
\end{tabular}

\subsection{Simulation}

Recently, a computer code based on the Monte Carlo method has been used for radiation dose calculation and radiation measurement system simulation. These computer codes are widely used because they are capable of 3D modeling and are continuously improving transport algorithms and nuclear data libraries. In this study, the thickness of a quantum dot-based plastic scintillator was optimized using MCNP and GEANT4. In addition, 30 and $50 \mathrm{~mm}$ thick quantum dot-based plastic scintillators were fabricated and tested using real radiation sources. EJ-200 of a commercial plastic scintillator was modeled with the same specifications as the scintillator purchased from Eljen technology Co. Ltd., and a plastic scintillator containing quantum dots was modeled by adding quantum dot material to the polystyrene material. The MCNP code was utilized to optimize the plastic thickness. The MCNP code is a radiation transmission code developed by Los Alamos National Laboratory. MCNP accounts for Compton scattering and Thomson scattering (coherent) for photons, fluorescence emission after photoelectric absorption, pair-production through local emission of annihilation radiation, and the bremsstrahlung process. The code simulates individual particle trajectories, and the stochastic events that make up the interaction process of nuclear particles with material are simulated sequentially. Each particle can create additional particles (daughters) or tracks at the point 
of impact. Each history (from birth to death) is considered an independent random event. Since MCNP includes a counting function for the energy accumulated in the detector, it is suitable for modeling the detector response. Thus, counting the pulse height per photon emitted from the source gives the absolute efficiency for each spectral peak. In the MCNP code, the tracing of each photon, caused by radiation interaction with the material, continues until the energy is low enough. GEANT4 is a tool for performing general-purpose nuclear and particle physics computational simulations based on C++. GEANT4 can be installed on Unix, Linux, and Windows operating systems, but the recommended operating system is Linux. GEANT4 is basically based on the Monte Carlo method. The Monte Carlo method is a method to solve statistical problems using random samples (0 to 1$)$ using random numbers. It is known to be effective in solving possible problems. The simulation conditions were set the same as MCNP conditions.

\subsection{Radiological Measurement}

The fabricated plastic scintillator was connected to a photomultiplier tube and a multichannel analyzer (MCA) for performance analysis, and a measurement experiment was performed using a ${ }^{137} \mathrm{Cs},{ }^{60} \mathrm{Co}$ disc source. The distance from the disc source was set to 20,50, and $100 \mathrm{~mm}$ to collect data, respectively, and the measurement efficiency was calculated. The measurement system consisted of a photomultiplier tube (ET-9266KB, ET-Enterprise Ltd.), a preamplifier (Amcrys 544, Amcrys), an amplifier (DT 5781, CAEN), and a multi-channel analyzer (DT 5781, CAEN). The signal generated by the plastic detector was amplified through a preamplifier and amplifier, and the amplified analog signal was digitized and stored through a multi-channel analyzer. In addition, a point source of ${ }^{137} \mathrm{Cs}$ and ${ }^{60} \mathrm{Co}$ was used to set the basic signal processing value of the plastic detector. Considering the half-life, the radioactivity of the ${ }^{137} \mathrm{Cs}$ and ${ }^{60} \mathrm{Co}$ point sources was $342.28 \mathrm{kBq}$ and $236.95 \mathrm{kBq}$, respectively.

\section{Results and Discussion}

\subsection{Characteristic Analysis of Plastic Scintillator Fabricated}

$\mathrm{CdS} / \mathrm{ZnS}$ quantum dots having an emission wavelength of 424-470 $\mathrm{nm}$, mentioned in Table 1, were loaded into a plastic matrix for the development of a high-performance plastic scintillator. Two computational simulation codes were used to optimize the thickness of the CdS/ZnS-loaded plastic scintillator. In addition, an actual plastic scintillator was fabricated, and measurement tests were also performed. Figure 1 shows monolith-type plastic scintillators fabricated. The diameters were the same for each at $50 \mathrm{~mm}$, and the thicknesses were 5,30 , and $50 \mathrm{~mm}$, and then optical properties and measurement tests were performed. Absorbance and transmittance of each of 5, 30, and $50 \mathrm{~mm}$ thick CdS/ZnSloaded plastics and commercial plastics were measured. Absorbance was measured using a lamp using a balanced deuterium and tungsten halogen source, as shown in Figure 2, and measured in the wavelength range of 200 to $1025 \mathrm{~nm}$ using a FLAME spectrometer. Figure 3 shows the absorbance and transmittance of $50 \mathrm{~mm}$ thickness. The same results were shown for plastic scintillators with thicknesses of 5 and $30 \mathrm{~mm}$. The transmittance of CdS/ZnS-loaded plastic was measured to be slightly higher than that of commercial plastic, which means that the quantum dot material was uniformly dispersed. The small difference in the results of commercial plastics and CdS/ZnS-loaded plastics is caused by the degree of scratches generated in the cutting and polishing processes.

In addition to absorbance, time-resolved fluorescence properties for three thicknesses were analyzed. The fluorescence characteristics of the prepared scintillator and commercial scintillator were analyzed using a time-resolved fluorescence spectrophotometer (Spectrofluorometer, HORIBA, Fluorolog-3). Figure 4a shows a schematic representation of the photoluminescence analysis regarding a real picture of the apparatus setup, and the path of the laser beam for analysis of the plastic scintillator is exhibited in Figure $4 \mathrm{~b}$. Figure $4 \mathrm{c}$ shows a sample compartment module of the plastic scintillator fabricated in this study. As the excitation light source, a $450 \mathrm{~W} \mathrm{CW}$ (continuous wave) ozone-free xenon arc 
(250-2500 nm) and a UV xenon light tube were used. Time-resolved fluorescence spectroscopy can measure fluorescence and phosphorescence in the UV-Vis-NIR region with high sensitivity for luminescent materials. It is a device that can measure the extinction time of fluorescence and phosphorescence down to the several pico (s) range. In addition, various holders are available to measure samples in the liquid, solid, or film state.

(a)

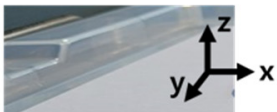

$5 \mathrm{~mm} \uparrow$

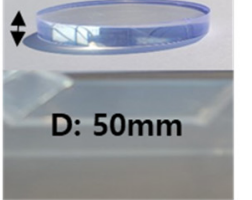

(b)

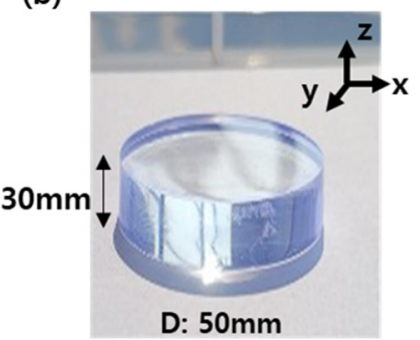

(c)

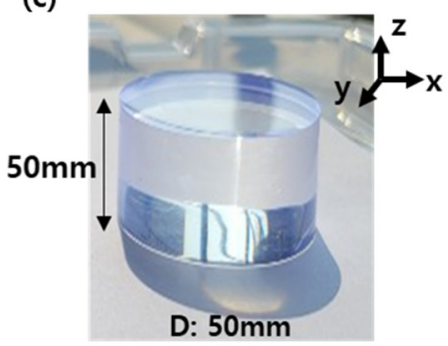

Figure 1. Different volume and thickness plastic scintillators: (a) $5 \mathrm{~mm}$ thickness; (b) $30 \mathrm{~mm}$ thickness; (c) $50 \mathrm{~mm}$ thickness.

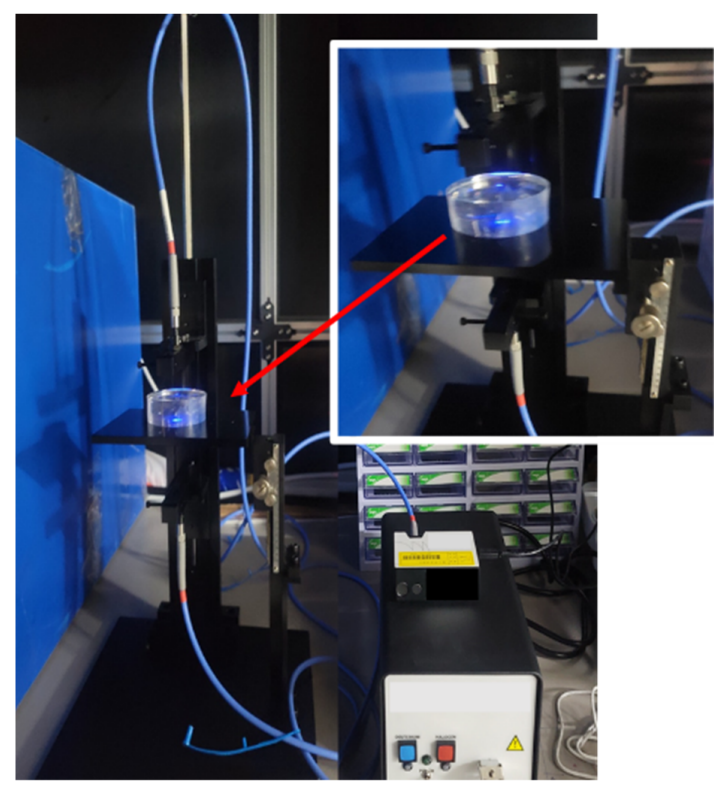

Figure 2. Apparatus setup for measurements of absorption and transmission based on fabricated plastic scintillator.

(a)

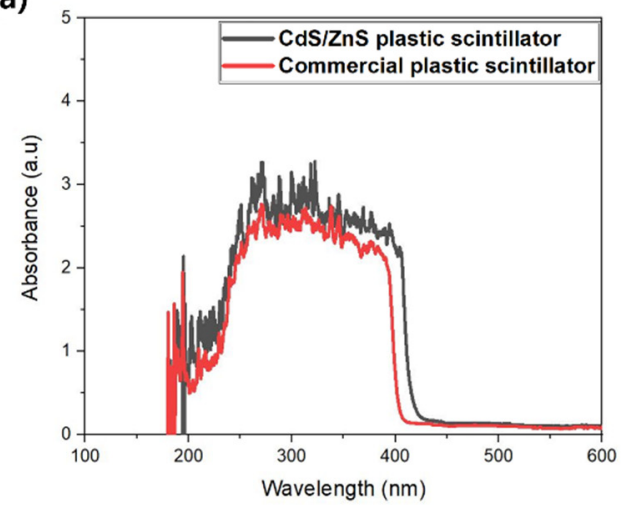

(b)

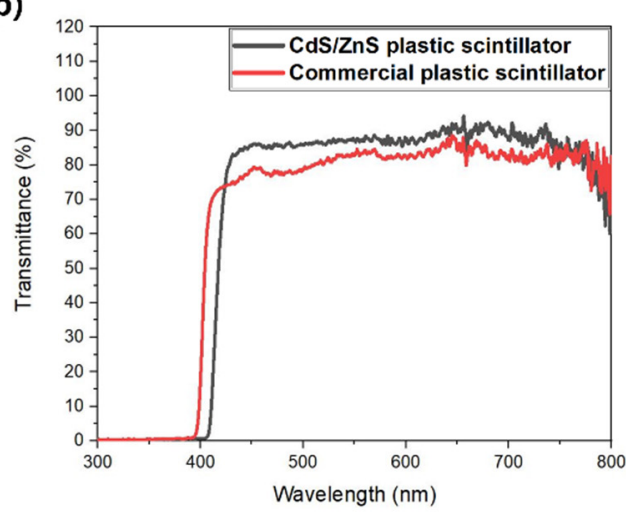

Figure 3. Absorbance (a) and transmittance (b) of commercial plastic and CdS/ZnS-loaded plastic scintillator fabricated with a thickness of $50 \mathrm{~mm}$. 
(a)

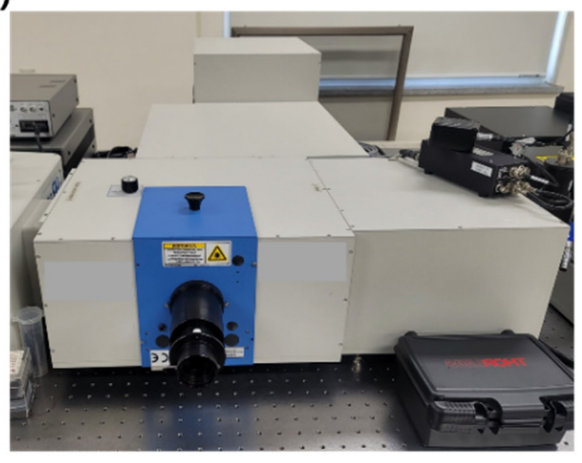

(b)

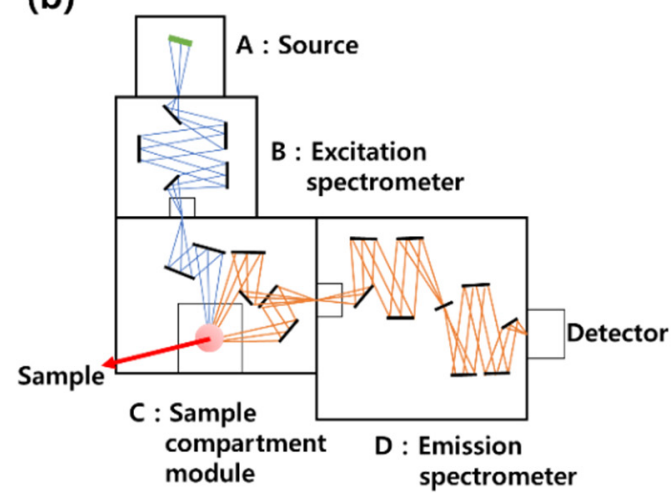

(c)

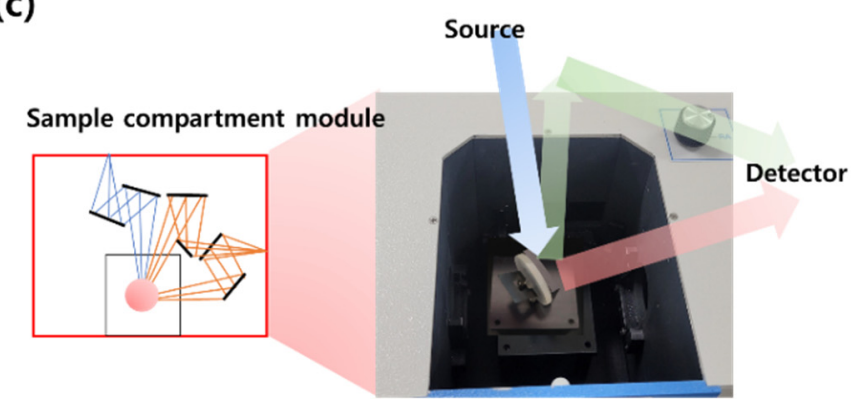

Figure 4. Schematic representation of the photoluminescence analysis: (a) real picture of apparatus setup; (b) path of the laser beam for analysis of plastic scintillator; (c) sample compartment module of plastic scintillator.

As shown in Figure 5a-c, it was performed on plastic scintillators with thicknesses of 5, 30 , and $50 \mathrm{~mm}$ for a photoluminescence analysis. Since the existing equipment was capable of a maximum thickness of $15 \mathrm{~mm}$, the sample holder was self-produced and utilized to enable a maximum thickness of $50 \mathrm{~mm}$. (Figure $5 \mathrm{a}-\mathrm{c}$ ) As a result, the CdS/ZnS-loaded plastic scintillator showed emission peaks at $459 \mathrm{~nm}$ and commercial plastic at $456 \mathrm{~nm}$. The emission peak can be seen in Figure 6a,b. As shown in Figure 6a,b, the emission peak showed the emission peak in the same wavelength band regardless of the thickness, and when the emission wavelength bands of the commercial scintillator and the quantum dotbased plastic were compared, it was observed that the emission wavelength was shifted by the quantum dot. The peak seen at about $500 \mathrm{~nm}$ in Figure 6a is considered to be caused by small defects such as scratches on the plastic scintillator surface.

(a)

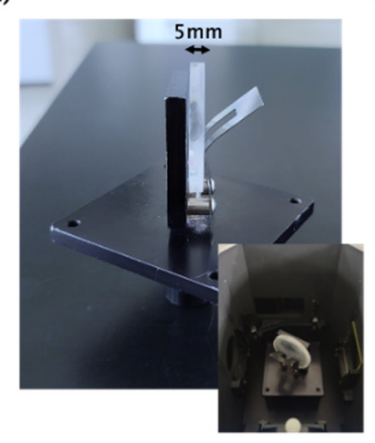

(b)

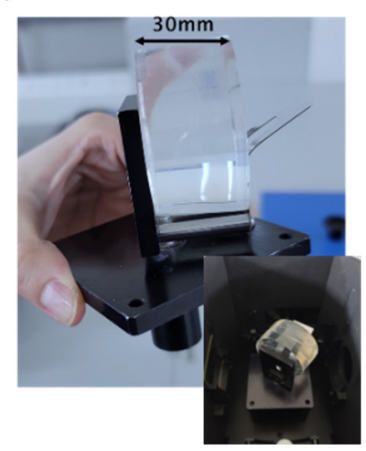

(c)

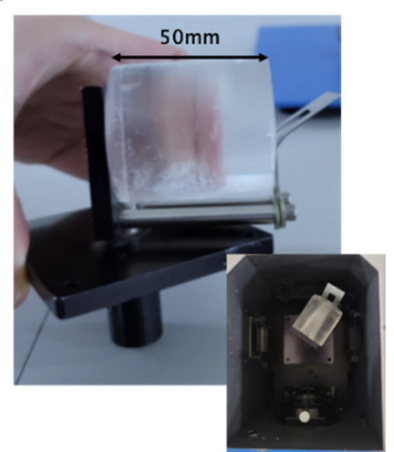

Figure 5. Holder with different spacer bars for photoluminescence analysis of plastic scintillator fabricated. (a) $5 \mathrm{~mm}$ thickness; (b) $30 \mathrm{~mm}$ thickness; (c) $50 \mathrm{~mm}$ thickness. 
(a)

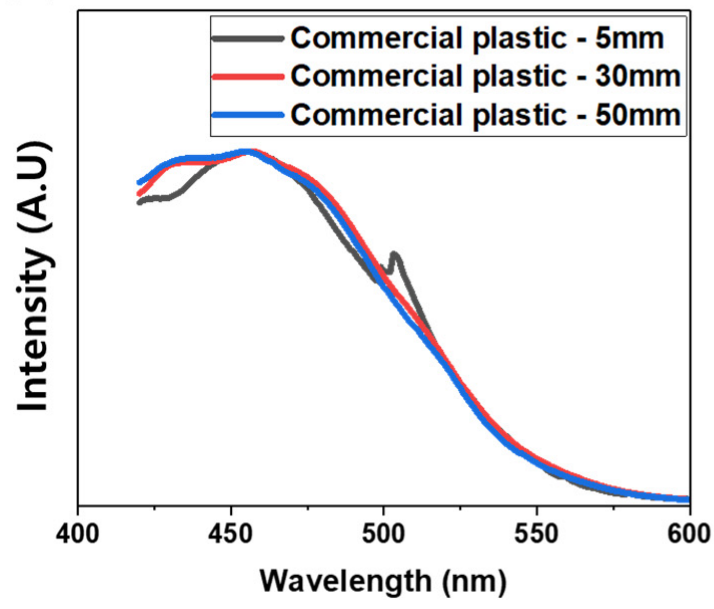

(b)

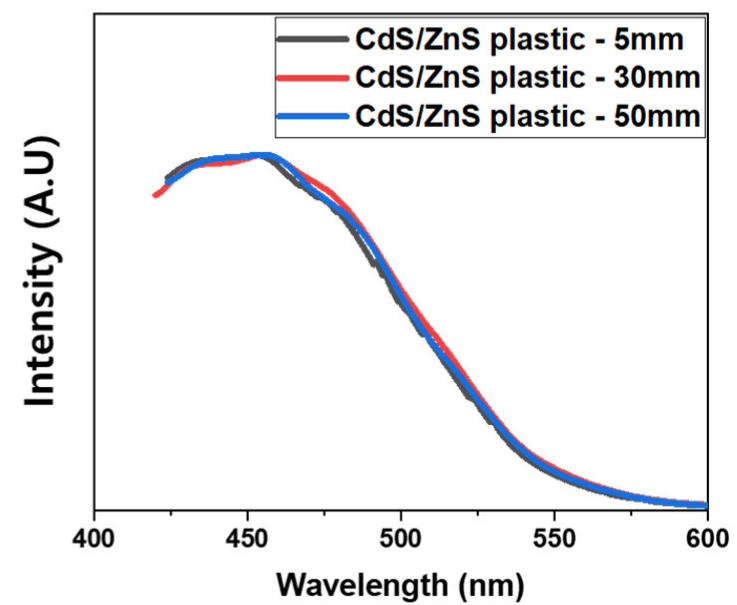

Figure 6. Photoluminescence analysis of (a) commercial plastic scintillator and (b) CdS/ZnS-based plastic scintillator.

\subsection{Simulations for Optimum Thickness of Plastic Scintillator}

A diameter of $50 \mathrm{~mm}$ and a thickness of 10 to $100 \mathrm{~mm}$ were simulated, and optical photons were set to be generated according to the Poisson distribution. The measurement results were compared with the results of two computer codes, and the characteristics of the computer codes used in this study are shown in Table 2.

Table 2. Comparison of characteristics of MCNP and GEANT4.

\begin{tabular}{c} 
Characteristics \\
\hline Simulation Codes \\
MCNP $\begin{array}{r}\text { The Monte Carlo N-Particle Transport Code (MCNP) was } \\
\text { developed by Los Alamos National Laboratory for the transport } \\
\text { of neutrons, photons, and electrons. }\end{array}$ \\
$\begin{array}{c}\text { It is applied in various fields such as radiation shielding, } \\
\text { dosimetry, radiography, and medical physics, and it is also used } \\
\text { in the design and analysis of the radiation measurement system. } \\
\text { It is a time-dependent code and was produced using Fortran } \\
\text { (425 Subroutines) and C. } \\
\text { GEANT4 is a computer simulation toolkit that tracks the } \\
\text { trajectory of particles as they pass through the material. } \\
\text { This code is used not only in high-energy physics, nuclear } \\
\text { physics, and accelerator physics, but also in medicine and } \\
\text { aerospace. } \\
\text { GEANT4 is designed with an object-oriented structure. } \\
\text { It is produced using C++ and consists of } 17 \text { basic classes. }\end{array}$ \\
\hline
\end{tabular}

Since there is no library for optical photons in MCNP, transport of photons cannot be considered. However, computational simulation considering electron transport or only gamma-ray transport is possible. It is possible to obtain energy deposited in the scintillator for each gamma-ray event by calculating the lost energy of gamma-rays or electrons using the pulse height tally (F8 tally). The geometry of the MCNP computational simulation of gamma-ray measurement is shown in Figure 7. The distances between the scintillation detector surface and the gamma-ray source were set to 20,50 , and $100 \mathrm{~mm}$, respectively, and $10^{9}$ gamma-rays were generated. For the energy of gamma-rays, computational simulations were performed with ${ }^{60} \mathrm{Co}$ and ${ }^{137} \mathrm{Cs}$ sources, respectively. 


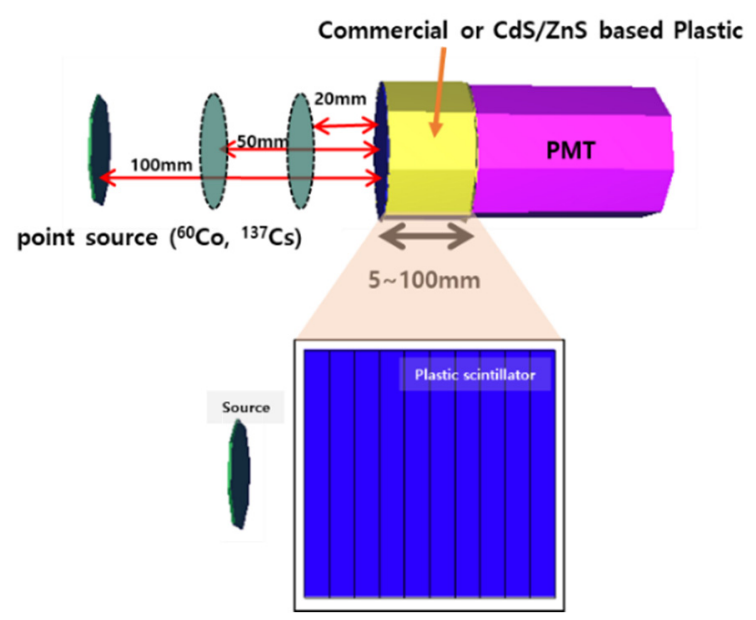

Figure 7. Geometry for application to MCNP.

In the case of GEANT4 code, there is a library for photons, so computational simulation considering the transport of photons is possible, and computational simulation considering the transport of electrons is also possible. Using GEANT4 code, computational simulation was performed, as shown in Figure 8. The energy of the photon was set as the energy of the ${ }^{60} \mathrm{Co}$ and ${ }^{137} \mathrm{Cs}$ sources, and $10^{9}$ gamma-rays were generated.

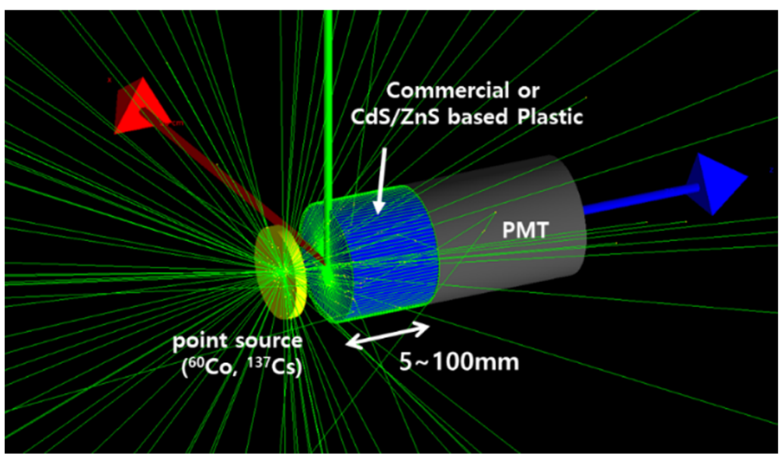

Figure 8. Geometry for application to GEANT4.

\subsection{Detection and Measurement of Gamma-Rays}

Since the plastic scintillator shows a continuous spectrum according to the detection characteristics, it was analyzed based on the Compton energy region. Compton edge energies of ${ }^{137} \mathrm{Cs}$ and ${ }^{60} \mathrm{Co}$ were calculated as 477.3 and $1040.79 \mathrm{keV}$, respectively. Compton edge energy of ${ }^{60} \mathrm{Co}$ was calculated as 963.20 and $1117.62 \mathrm{keV}$ based on 1173 and $1332 \mathrm{keV}$, which are photopeak energies. However, the plastic scintillator shows only one peak at an energy of about $1040.79 \mathrm{keV}$ due to its low resolution. In addition, in order to remove the afterglow of PMT generated during the plastic replacement process, the scintillator was stored in a dark room for about $12 \mathrm{~h}$ after replacement, and then a measurement experiment was performed. Figure 9 shows the measuring system configured for performance evaluation of the plastic scintillator.

Figure 10 shows the experimental and computational simulation results of 30 and $50 \mathrm{~mm}$ thick plastic scintillators, showing the results near the Compton edge. For comparison of results, thickness optimization was also performed on a commercial plastic scintillator, and the commercial plastic used in this study was EJ-200 purchased from EJ-technology. Figure $10 \mathrm{a}-\mathrm{d}$ shows the measurement results for ${ }^{60} \mathrm{Co}$ and ${ }^{137} \mathrm{C}$ s sources of commercial plastic scintillators, and Figure $10 \mathrm{e}-\mathrm{h}$ shows the measurement results for ${ }^{60} \mathrm{Co}$ and ${ }^{137} \mathrm{Cs}$ sources of $\mathrm{CdS} / \mathrm{ZnS}$-loaded plastic scintillators. 


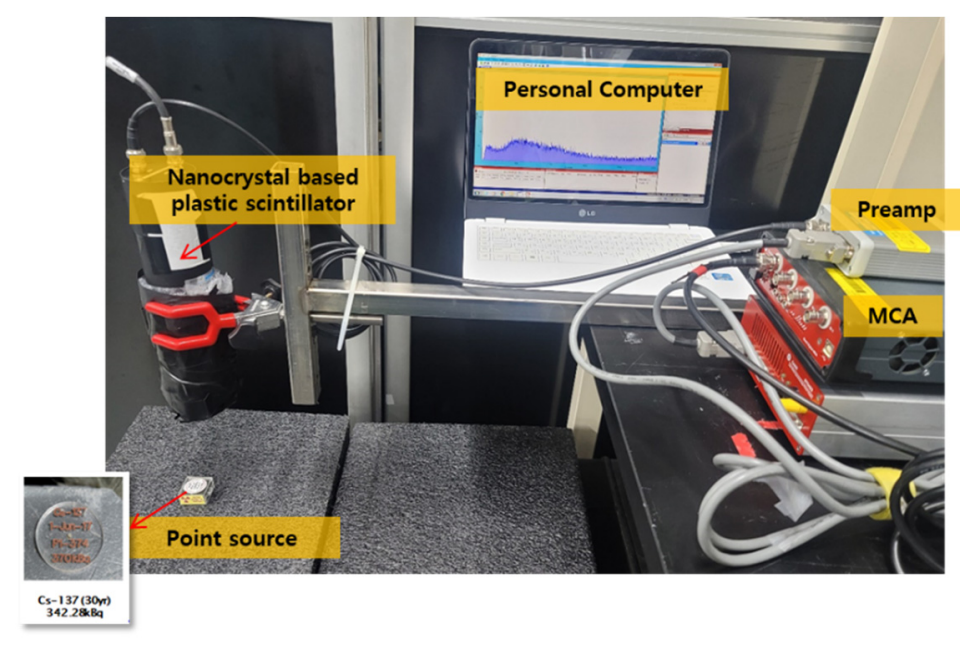

Figure 9. System configured for measurement by thickness of plastic scintillator.

(a)

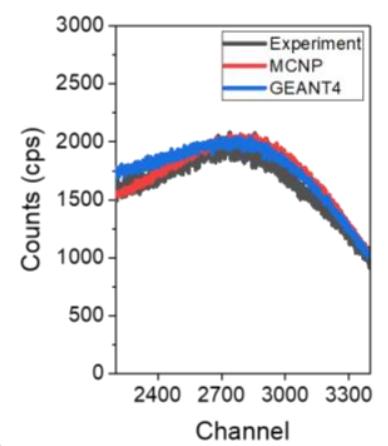

(c)

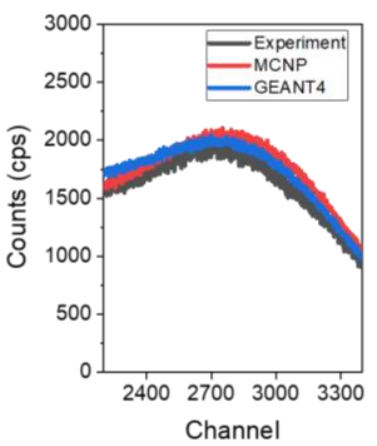

(e)

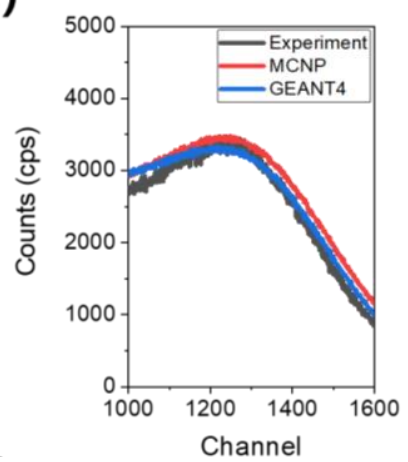

(b)

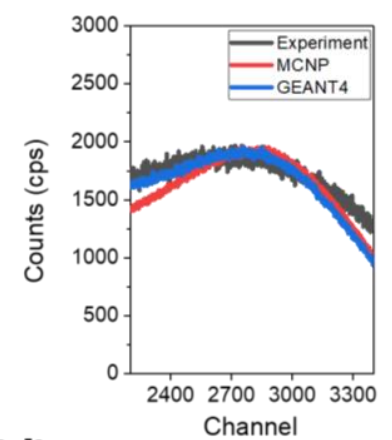

(d)

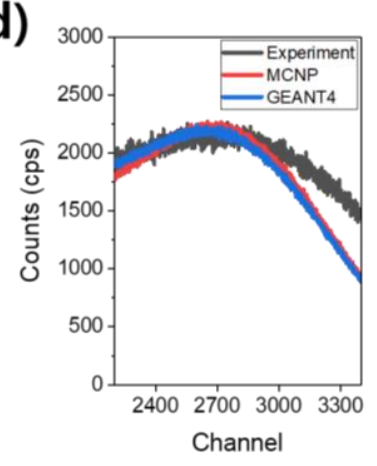

(f)

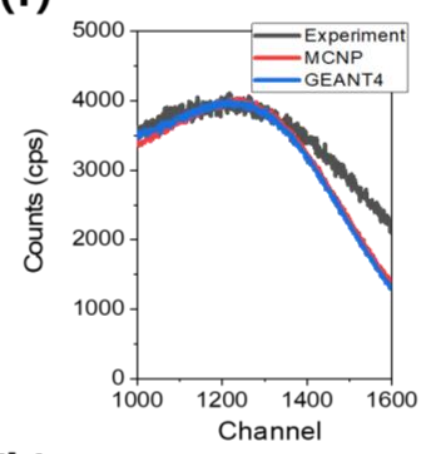

Figure 10. Cont. 
(g)

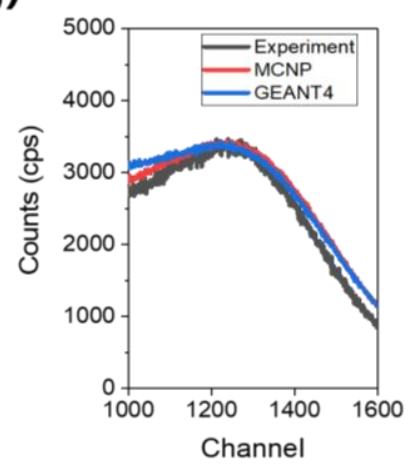

(h)

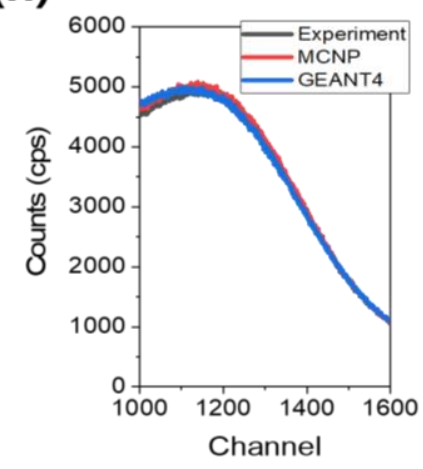

Figure 10. Experimental results and computational simulation results of commercial and CdS/ZnSloaded plastic scintillators: (a) $30 \mathrm{~mm}$ commercial plastic scintillator- ${ }^{60} \mathrm{Co}$; (b) $50 \mathrm{~mm}$ commercial plastic scintillator- ${ }^{60} \mathrm{Co}$; (c) $30 \mathrm{~mm} \mathrm{CdS} / \mathrm{ZnS}$-loaded plastic scintillator- ${ }^{60} \mathrm{Co}$; (d) $50 \mathrm{~mm} \mathrm{CdS} / \mathrm{ZnS}$ loaded plastic scintillator- ${ }^{60} \mathrm{Co}$; (e) $30 \mathrm{~mm}$ commercial plastic scintillator- ${ }^{137} \mathrm{Cs}$; (f) $50 \mathrm{~mm}$ commercial plastic scintillator_- ${ }^{137} \mathrm{Cs}$; (g) $30 \mathrm{~mm} \mathrm{CdS/ZnS-loaded} \mathrm{plastic} \mathrm{scintillator-}{ }^{137} \mathrm{Cs}$; (h) $50 \mathrm{~mm}$ $\mathrm{CdS} /$ ZnS-loaded plastic scintillator- ${ }^{137} \mathrm{Cs}$.

As shown in Figure 10, both commercial plastics and quantum dot-loaded plastics showed peaks at the Compton edge and were observed as spectra of the same trend.

The reason for the small difference between the simulation result and the actual measured value in the spectrum in Figure 10 is an error caused by not reflecting the actual measurement environment in the simulation geometry. In the Supplementary Materials, as shown in Figures S1 and S2, input data of the MCNP 6 simulation are presented using a CdS/ZnS plastic scintillator to ${ }^{60} \mathrm{Co}$ and ${ }^{137} \mathrm{Cs}$ sources. Furthermore, input data of the GEANT4 simulation are also presented using the CdS/ZnS plastic scintillator including construction and mac file to ${ }^{60} \mathrm{Co}$ and ${ }^{137} \mathrm{C}$ s sources in Figures S3-S6.

Figure 11 shows the error of total count that occurs whenever the thickness of the plastic scintillator increases by $10 \mathrm{~mm}$. The error is calculated based on the simulation results and is calculated using Equation (2).

$$
\text { Error of total } \operatorname{count}(\%)=\frac{\text { Total counts of }(x-10 \mathrm{~mm}) \text { plastic scintillator }}{\text { Total counts of }(x \mathrm{~mm}) \text { plastic scintillator }} \times 100
$$

(a)

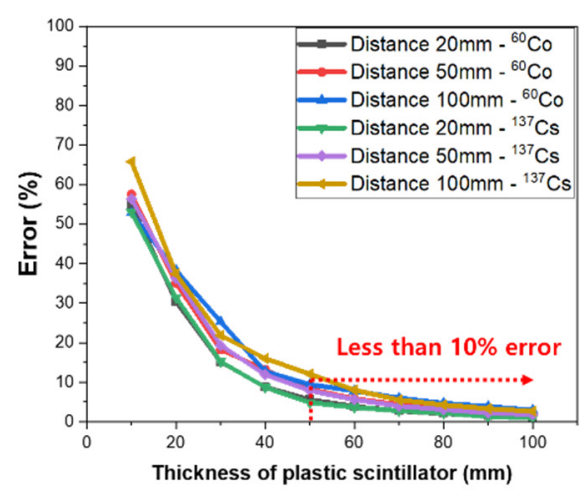

(b)

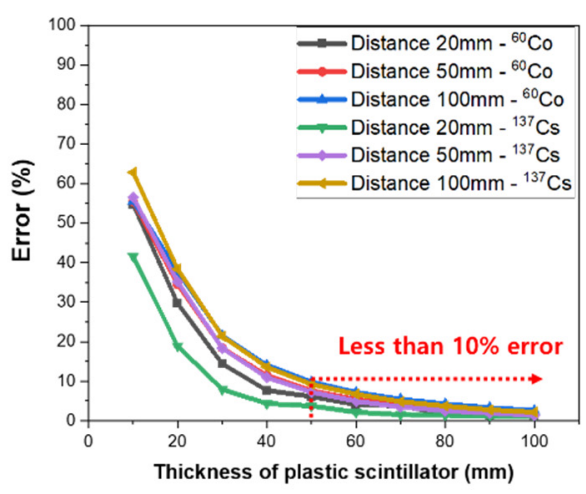

Figure 11. Error caused by increasing the plastic thickness (\%): (a) commercial plastic scintillator; (b) CdS/ZnS-loaded plastic scintillator.

Here, $x$ means the thickness of the plastic scintillator to be evaluated. The computational simulation was performed for each thickness of the plastic scintillator from 10 to $100 \mathrm{~mm}$ at intervals of $10 \mathrm{~mm}$. After calculating the total counts for each thickness, the difference 
in total counts when the thickness is increased by $10 \mathrm{~mm}$ is shown in Figure 11. Figure 11a is the result for a commercial plastic scintillator, and Figure $11 \mathrm{~b}$ is the result for a plastic scintillator loaded with CdS/ZnS. In Figure 11, it was confirmed that the difference in total counts was less than $10 \%$ for plastic scintillator thicknesses of $50 \mathrm{~mm}$ or more, which means an optimized thickness for high-efficiency gamma-ray detection, such as radioactive ${ }^{137} \mathrm{Cs}$ and ${ }^{60} \mathrm{Co}$. The larger and thicker the scintillator, the higher the total counts. However, due to the thick plastic scintillator, the generated scintillation cannot be transmitted to the PMT, which may have an adverse effect. Furthermore, Figure 12 shows the result of comparing the simulation and experimental measurement. Figure 12a is the result of a commercial plastic scintillator, and Figure 12b is the result of a plastic scintillator loaded with CdS/ZnS; $5 \mathrm{~mm}$ was too thin to be used for gamma measurement, so measurement experiments were not performed. Measurement experiments were only performed on plastic scintillators with thicknesses of 30 and $50 \mathrm{~mm}$. As the distance from the source increased, the total count decreased, but it could be observed that the total count of the CdS/ZnS-loaded plastic scintillator was higher than that of the commercial plastic scintillator. This was considered the effect of quantum dots.

(a)

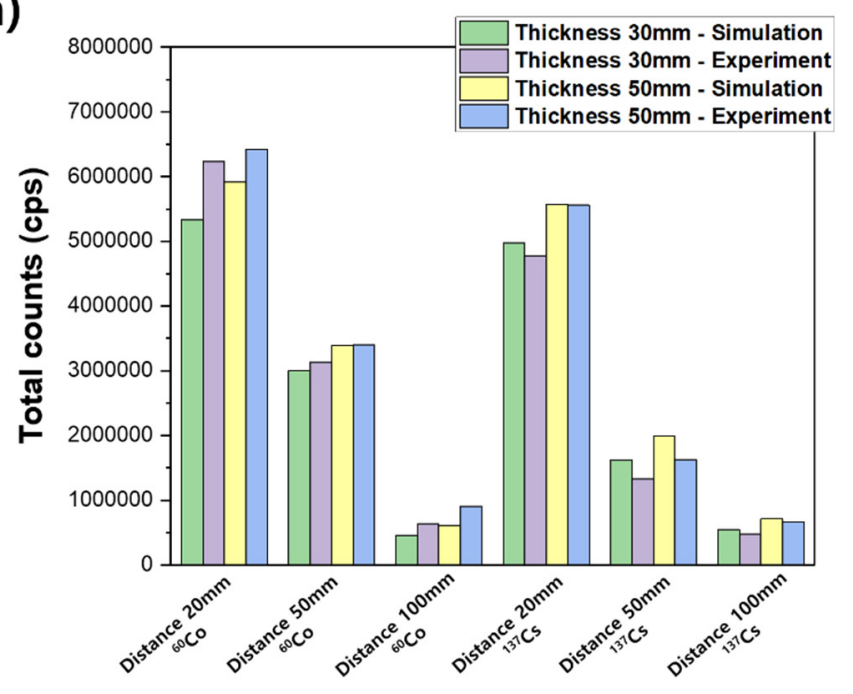

(b)

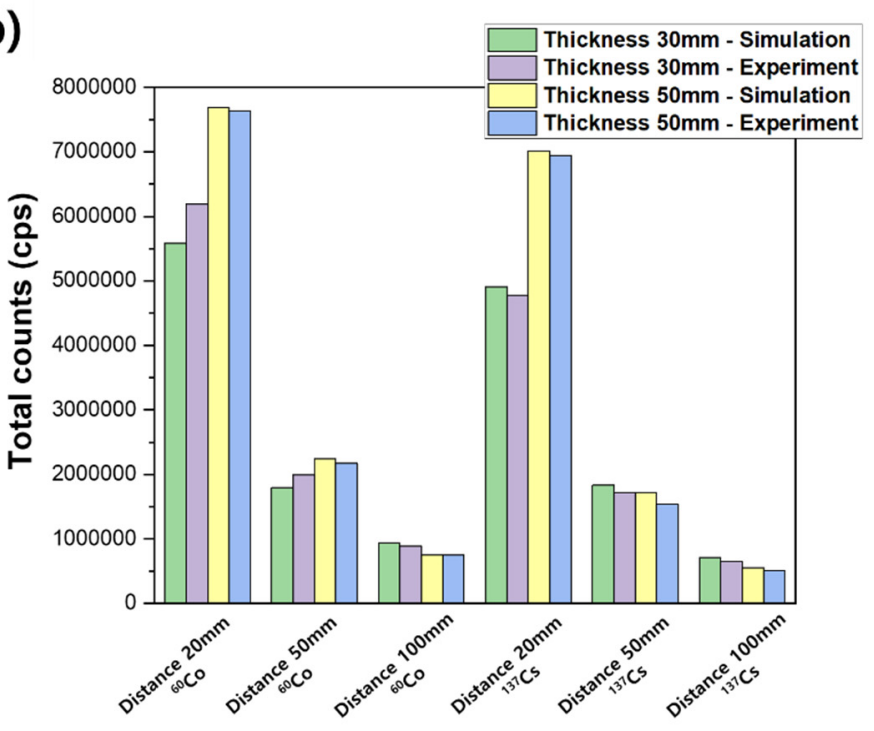

Figure 12. Total counts and errors of $30 \mathrm{~mm}$ and $50 \mathrm{~mm}$ calculated using GEANT4: (a) commercial plastic scintillator; (b) CdS/ZnS-loaded plastic scintillator. 


\section{Conclusions}

Simulated results along with experimental data were discussed for the optimization of a plastic scintillator for the detection of gamma-rays. The thickness of the quantum dot-based plastic scintillator for gamma measurement was optimized to $50 \mathrm{~mm}$. For plastic scintillator thickness optimization, MCNP and GEANT4 codes were used, and a series of interactions in the scintillator from the emissions of gamma-rays due to the decay of the radiation source was simulated. The simulation results of MCNP and GEANT4 were analyzed identically, and the total counts increased as the scintillator thickness increased. Since the shift of the Compton edge does not occur as the thickness increases, it means that even with a thin thickness of $10 \mathrm{~mm}$, all the ultraviolet rays emitted from polystyrene can be absorbed and converted into visible light, and the amount of light emitted is the same. In addition, as a result of measuring the fluorescence intensity of 30 and $50 \mathrm{~mm}$ thick plastics, it was confirmed that they were emitted in the same wavelength band. As a result of measurement and simulation, as the thickness of the plastic increases, the energy deposited increases, but if the thickness is too thick, it is difficult for the light from the scintillator to reach the photo sensor. In addition, as the thickness of the scintillator increases, the resolution decreases. The thickness of the scintillator is proportional to the amount of energy deposited, but it can affect the afterglow. Once the thickness of the scintillator is too thin, it is highly likely that incident photons pass through without interacting with the scintillator. In this study, it was confirmed that there was no significant difference in the amount of energy deposited at $50 \mathrm{~mm}$ or more, and it was confirmed that the error was small in the actual measurement and simulation results (less than 10\% error). The measurement results using the 30 and $50 \mathrm{~mm}$ thick scintillators were analyzed with the same trend as the simulation results.

It is important to optimize the size of the scintillator economically, and since the optical properties change when the material of the quantum dot is changed, thickness optimization of the plastic scintillator is essential. When optimizing the thickness, the gamma-ray energy and background should be considered. In this study, thickness optimization was performed using two computational simulation codes, and the errors between actual measurement results and computational simulation results were analyzed to be less. In the future, it is planned to manufacture after optimizing the thickness of plastic loaded with various quantum dot materials through computational simulation. Finally, an optimized plastic scintillator for the detection of radioactive cesium and cobalt was elucidated. A monolith-typed optimized plastic scintillator was fabricated, and it was compared with the performance of a commercial plastic scintillator. The highly reliable and direct detection of radioactive cesium and cobalt has gained potential interest due to in situ detection and monitoring in environments. The understanding of an improved and advanced plastic scintillator could pave the way for improving detection technology or to suggest the development of new materials with superior properties.

Supplementary Materials: The following are available online at https:/ / www.mdpi.com/article/ 10.3390/chemosensors9090239/s1, Figure S1: Input data of MCNP 6 simulation (CdS/ZnS plastic scintillator— ${ }^{60}$ Co source); Figure S2: Input data of MCNP 6 simulation (CdS/ZnS plastic scintillator${ }^{137}$ Cs source); Figure S3: Input data of GEANT4 simulation (CdS/ZnS plastic scintillator-Construction file); Figure S4: Input data of GEANT4 simulation (CdS/ZnS plastic scintillator-Run file); Figure S5: Input data of GEANT4 simulation (CdS/ZnS plastic scintillator-Source $\left({ }^{60} \mathrm{Co}\right)$ mac file); Figure 66 : Input data of GEANT4 simulation $\left(\mathrm{CdS} / \mathrm{ZnS}\right.$ plastic scintillator-Source $\left({ }^{137} \mathrm{Cs}\right)$ mac file).

Author Contributions: Conceptualization and original draft preparation: S.M.; experimental setup and simulation: Y.K., K.-H.K. and B.S.; supervision and editing C.R., S.H. and J.C. All authors have read and agreed to the published version of the manuscript.

Funding: This research received no external funding.

Institutional Review Board Statement: Not applicable.

Informed Consent Statement: Not applicable. 
Data Availability Statement: Data available on request from the authors.

Acknowledgments: This work was supported by the Nuclear Research and Development Program through the National Research Foundation (NRF) of Korea funded by the Ministry of Science and ICT (No. 2017M2A8A5015143).

Conflicts of Interest: The authors declare no conflict of interest.

\section{References}

1. Schorr, M.G.; Torney, F.L. Solid non-crystalline scintillation phosphors. Phys. Rev. 1950, 80, 474-475. [CrossRef]

2. Buck, W.L.; Swank, R.K. Efficient Plastic Scintillators. Nucleonics 1953, 11, 48-52.

3. Hayes, F.N.; Ott, D.G.; Kerr, V.N. Pulse height comparison of primary solutes. Nucleonics 1955, 13, 38-41.

4. Ott, D.G.; Hayes, O.F.; Hansbury, E.; Kerr, V.N. Liquid scintillators. V. Absorption and Fluorescence spectra of 2,5-Diaryloxazoles and related compounds. J. Am. Chem. Soc. 1957, 79, 5448-5454. [CrossRef]

5. Hajagos, T.J.; Liu, C.; Cherepy, N.J.; Pei, Q. High Z Sensitized plastic scintillators: A review. Adv. Mater. 2018, $30,1706956$. [CrossRef]

6. Murugadoss, G.; Rajesh Kumar, M. Optical and structural characterization of CdS/ZnS CdS:Cu${ }^{2+} / \mathrm{ZnS}$ core-shell nanoparticles. Luminescence 2014, 29, 663-668. [CrossRef]

7. Youn, H.C.; Baral, S.; Fendler, J.H. Dihexadecyl phosphate, vesiclestabilized and in situ generated mixed cadmium sulfide and zinc sulfide semiconductor particles: Preparation and utilization for photosensitized charge separation and hydrogen generation. J. Phys. Chem. 1988, 92, 6320-6327. [CrossRef]

8. Kortan, A.R.; Hull, R.; Opila, R.L.; Bawendi, M.G.; Steigerwald, M.L.; Carroll, P.J.; Brus, L.E. Nucleation and Growth of CdSe on ZnS Quantum crystallite seeds, and Vice versa, in Inverse Micelle media. J. Am. Chem. Soc. 1990, 112, 1327-1332. [CrossRef]

9. Danek, M.; Jensen, K.F.; Murray, C.B.; Bawendi, M.G. Synthesis of luminescent thin-film CdSe/ZnSe quantum dot composites using CdSe quantum dots passivated with an overlayer of ZnSe. Chem. Mater. 1996, 8, 173-180. [CrossRef]

10. Littau, K.A.; Szajowski, P.J.; Muller, A.J.; Kortan, A.R.; Brus, L.E. A luminescent silicon nanocrystal colloid via a high-temperature aerosol reaction. J. Phys. Chem. 1993, 97, 1224-1230. [CrossRef]

11. Wilson, W.L.; Szajowski, P.J.; Brus, L.E. Quantum confinement in size-selected, surface-oxidized silicon nanocrystals. Science 1993 262, 1242-1244. [CrossRef]

12. Hines, M.A.; Guyot-Sionnest, P. Synthesis and characterization of strongly luminescing ZnS-capped CdSe nanocrystals. J. Phys. Chem. 1996, 100, 468-471. [CrossRef]

13. Chang, K.S. Structural and Optical Characteristics of ZnS/CdS powders and thin films. J. Korean Inst. Mil. Sci. Technol. 2010, 13, 659-664.

14. Rajakrishna, K.; Dhanasekaran, A.; Yuvaraj, N.; Ajoy, K.C.; Venkatraman, B. Effect of high Z materials loading in the performance of polystyrene-based thin-film plastic scintillators. Nucl. Instrum. Methods Phys. Res. Sect. A 2021, 1008, 165454. [CrossRef]

15. Kagami, K.; Koshimizu, M.; Fujimoto, Y.; Kishimoto, S.; Haruki, R.; Nishikido, F.; Asai, K. X-ray detection properties of Bi-loaded plastic scintillators synthesized via solvent evaporation. Radiat. Meas. 2020, 135, 106361. [CrossRef]

16. Kim, D.G.; Lee, S.M.; Park, J.S.; Son, J.B.; Kim, T.H.; Kim, Y.H.; Pak, K.H.; Kim, Y.K. Performance of 3D printed plastic scintillators for gamma-ray detection. Nucl. Eng. Technol. 2020, 52, 2910-2917.

17. Posar, J.A.; Davis, J.; Brace, O.; Sellin, P.; Griffith, M.J.; Dhez, O.; Wilkinson, D.; Lerch, M.L.F.; Rosenfeld, A.; Petasecca, M. Characterization of a plastic dosimeter based on organic semiconductor photodiodes and scintillator. Phys. Imaging Radiat. Oncol. 2020, 14, 45-52. [CrossRef]

18. Ji, Y.Y.; Ochi, K.; Hong, S.B.; Nakama, S.; Sanada, Y.; Mikami, S. Performance of in situ gamma-ray spectrometry in the assessment of radioactive cesium deposition around the Fukushima Daiichi nuclear power plant. Radiat. Phys. Chem. 2021, $179,109205$. [CrossRef]

19. Lee, C.K.; Park, S.W.; Kim, H.R. Development of mobile scanning system for effective in-situ spatial prediction of radioactive contamination at decommissioning sites. Nucl. Instrum. Methods Phys. Res. Sect. A 2020, 966, 163833. [CrossRef]

20. Dufour, N.; Dumazert, J.; Barat, E.; Bertrand, G.H.V.; Carrel, F.; Dautremer, T.; Laine, F.; Sari, A. Measurement of low-activity uranium contamination by gamma-ray spectrometry for nuclear decommissioning. Nucl. Instrum. Methods Phys. Res. Sect. A 2020, 951, 162976. [CrossRef]

21. Amgarou, K.; Aspe, F.; Idoeta, R.; Herranz, M. Recommendations for the selection of in situ measurement techniques for radiological characterization in nuclear/radiological installations under decommissioning and dismantling processes. Prog. Nucl. Energy 2021, 137, 103761. [CrossRef]

22. Bae, J.W.; Kim, H.R. Plastic scintillator beta ray scanner for in-situ discrimination of beta ray and gamma ray radioactivity in soil. Nucl. Eng. Technol. 2020, 52, 1259-1265. [CrossRef]

23. Knoll, G.F. Chapter 8 Scintillation Detector Principles. Radiation Detection and Measurement, 4th ed.; John Wiley \& Sons, Inc.: New York, NY, USA, 2010; pp. 220-247.

24. Xiao, B.; Lee, S.; Hagel, K.; Haddad, F.; Li, J.; Lou, Y.; Mdeiwayeh, N.; Tezkratt, R.; Wada, R.; Utley, D.; et al. Production and thickness determination of thin plastic scintillator foils. Nucl. Instrum. Methods Phys. Res. Sect. A 1995, 355, 258-260. [CrossRef] 
25. Ros, G.; Saez-Cano, G.; Medina-Tanco, G.A.; Supanitsky, A.D. On the design of experiments based on plastic scintillators using GEANT4 simulations. Radiat. Phys. Chem. 2018, 153, 140-151.

26. Kim, K.H. Fabrication and a Study on the Tapered Scintillator Radiation Sensors. Master's Thesis, Jeju National University, Jeju, Korea, December 2003.

27. Moszynski, M.; Kapusta, M.; Mayhugh, M.; Wolski, D.; Flyckt, S.O. Absolute light output of scintillators. IEEE Trans. Nucl. Sci. 1997, 44, 1052-1061. [CrossRef]

28. Kim, J.W.; Lee, B.C.; Uhm, Y.R.; Miller, W.H. Enhancement of thermal neutron attenuation of nano-B ${ }_{4}$ C, - BN dispersed neutron shielding polymer nanocomposites. J. Nucl. Mater. 2014, 453, 48-53. [CrossRef]

29. Kang, J.K.; Lee, J.O.; Lee, D.J. Calculation of Dose Distribution for SBRT Patient Using Geant4 Simulation Code. Prog. Med. Phys. 2015, 26, 36-41. [CrossRef]

30. Sung, S.H.; Kim, H.R. Optimization of airborne alpha beta detection system modeling using MCNP simulation. Nucl. Eng. Technol. 2020, 52, 841-845. [CrossRef]

31. Childress, N.L.; Miller, W.H. MCNP analysis and optimization of a triple crystal phoswich detector. Nucl. Instrum. Methods Phys. Res. Sect. A 2002, 490, 263-270. [CrossRef]

32. Tam, H.D.; Yen, N.T.H.; Tran, L.B.; Chuong, H.D.; Thanh, T.T. Optimization of the Monte Carlo simulation model of NaI(Tl) detector by Geant4 code. Appl. Radiat. Isot. 2017, 130, 75-79.

33. Yehuda-Zada, Y.; Pritchard, K.; Ziegler, J.B.; Cooksey, C.; Siebein, K.; Jackson, M.; Hurlbut, C.; Kadmon, Y.; Cohen, Y.; Ibberson, R.M.; et al. Optimization of 6LiF:ZnS(Ag) scintillator light yield using GEANT4. Nucl. Instrum. Methods Phys. Res. Sect. A 2018, 892, 59-69. [CrossRef]

34. Nam, J.S.; Kim, Y.E.; Hong, S.B.; Seo, B.K.; Kim, K.H. Performance Evaluation of a Plastic Scintillator for Making a In-situ Beta Detector. New Phy. SaeMulli 2017, 67, 1080-1085.

35. Kang, H.R.; Min, S.J.; Seo, B.K.; Roh, C.H.; Hong, S.B.; Cheong, J.H. Preliminary Studies of Perovskite-Loaded Plastic Scintillator Prototypes for Radioactive Strontium Detection. Chemosensors 2021, 9, 53. [CrossRef]

36. Min, S.J.; Kang, H.R.; Seo, B.K.; Roh, C.H.; Hong, S.B.; Cheong, J.H. Integrated and Portable Probe Based on Functional Plastic Scintillator for Detection of Radioactive Cesium. Appl. Sci. 2021, 11, 5210. [CrossRef]

37. Healy, M.S.; Hanson, J.E. Fluorescence excitation spectroscopy of polystyrene near the critical concentration c*. J. Appl. Polym. Sci. 2007, 104, 360-364. [CrossRef]

38. Park, J.M.; Kim, H.H.; Hwang, Y.S.; Kim, D.H.; Park, H.W. Scintillation properties of quantum-dot doped styrene based plastic scintillators. J. Lumin. 2014, 146, 157-161. [CrossRef]

39. Madkour, M.; Salih, T.; Al-Sagheer, F.; Bumajdad, A. Nano heterostructured photo-stable $\mathrm{CdZn}_{1-\mathrm{x}} \mathrm{S}$ heterojunction as a nonphotocorrosive visible light active photocatalyst. Opt. Mater. Express 2016, 6, 2857-2870. [CrossRef]

40. Xinmei, L.; Yang, J.; Xinzheng, L.; Yugang, Z.; Shanying, L.; Junwei, L.; Tingting, H.; Binbin, W.; Honghai, Z. Highly luminescent blue emitting CdS/ZnS core/shell quantum dots via a single-molecular precursor for shell growth. Mater. Chem. Phys. 2011, 130, 909-914. 